\title{
Preliminary Evaluation of Coating Hydrophobization of Natural Stone from Repedea - Iasi area (Romania)
}

\author{
VASILE PELIN ${ }^{1}$, IRINA RADINSCHI ${ }^{2}$, VASILICA CIOCAN ${ }^{3}$, ION SANDU ${ }^{4,5}$, TUDOR BOGDAN COMAN TH $^{2}$ \\ MARIUS MIHAI CAZACU2* \\ ${ }^{1}$ Alexandru Ioan Cuza University of Iasi, Faculty of Geography and Geology, Environmental Sciences Department \& Faculty \\ of Physics, LOASL Laboratory, 11 Carol I Blvd., 700506, Iasi, Romania \\ ${ }^{2}$ Gheorghe Asachi Technical University of Iasi, Department of Physics, 67 Prof. Dimitrie Mangeron Blvd., 700050, Iasi, \\ Romania \\ ${ }^{3}$ Gheorghe Asachi Technical University of Iasi, Faculty of Civil Engineering and Building Services, 1 Prof. Dimitrie \\ Mangeron Blvd., 700050, Iasi, Romania \\ ${ }^{4}$ Alexandru Ioan Cuza University of Iasi, Institute of Interdisciplinary Research - Sciences Department, ARHEOINVEST \\ Center, 11 Carol I Blvd., 700506, Iasi, Romania \\ ${ }^{5}$ Romanian Inventors Forum, 3 Sf. Petru Movila Str., 700089, Iasi, Romania
}

Given the need for the development of interdisciplinary studies and research, including in the Engineering field and in the Building sector, this paper suggests a multidisciplinary approach to the maintenance of lithic surfaces, by analyzing some high-performance building materials and products, such as hydrophobization coating films. As it has been determined and acknowledged by the scientific community worldwide, the main aggression factor affecting the geomaterials used in the construction of heritage assets is represented by water and/or excessive humidity, especially in the case of the use of porous limestone, such as those available in the Repedea - Iasi area (Romania). Therefore, in order to ensure better maintenance of a lithic calcareous material of this type, it is necessary to use a hydrophobic preventive treatment. The findings of the research conducted and described in this paper represent one of the first steps on the path to standardization regarding the confirmation of the efficiency of the hydrophobization products, which have been increasingly present on the Romanian specialized market lately. Thus, this paper is addressed to all the specialists interested in maintaining the lithic surfaces exposed to various environmental factors, as it is useful for both historical and civil constructions.

Keywords: stone hydrophobization, coating products, UV exposure, stone aging, historical buildings, conservation Science

Although natural stone is considered a construction material that stands the test of time, the degradation and deteriorate rate of architectural surfaces has been increasing over the last decades, especially due to anthropic causes, mainly to atmospheric pollution [1-3], but also due to intrinsic factors, by using low quality indigenous lithic materials, which favor the rapid onset of the aforementioned alterations. Such a situation is often encountered in the case of numerous historical buildings in Iasi area, erected between the XVth and the XIXth centuries, as well as during the first decades of the twentieth century, the most sought after building stones being the porous limestone in the Scheia, Pietrarie and Paun Repedea areas located south of Iasi City.

For the stone constructions in the city of Iasi, most of the building materials were found in the surrounding areas of the city [4], where the geological context allowed the formation of porous sedimentary rock deposits [5, 6]. Therefore, as lithic materials used in constructions, sedimentary rocks in Iasi area have relatively low strength and durability [7-10], and for this reason their use in the maintenance and/or restoration processes of historical buildings requires a series of in-depth studies and research, with reference to the choice of stone and the development of an appropriate protocol that clarifies a number of issues, such as [11]:

a) spot/area in the building requiring restoration works;

b) extent of current (active) damages and degradations;

*email: cazacumarius@gmail.com 
c) compatibility with already existing lithic materials;

d) anticipation of the occurrence of possible adverse effects.

Knowledge about the behavior of materials in the processes of consolidation, impregnation or coating is the focus of any specialist in the field of periodic maintenance of buildings or historical monument preservation and restoration. Whatever the chosen material, it must be thoroughly studied from the point of view of its reactivity and compatibility with the support stone substrates, particularly as concerns both the interactions between material and support, and those in time, under the action of exogenous factors, such as natural light, which it may also cause undesirable results, in the case of hydrophobization, by possible color changes of the surfaces undergoing treatment, under the influence of UV radiation [12].

As the number of consolidation products, impregnation or coating materials currently available on the market is quite high, this paper describes a method of preliminary evaluation of several hydrophobization agents for natural stone, by artificial ageing of coated surfaces, using UV rays, under laboratory conditions.

\section{Experimental part}

\section{Materials and methods}

This paper describes a method that assesses hydrophobization efficiency, under laboratory conditions, for eight types of coating agents, involving both colorimetric analysis of the treated lithic surfaces, both before and after coating, and during the accelerated artificial ageing by exposure to UV radiation, also under laboratory conditions. The evaluation was performed by periodic colorimetric monitoring and by comparing the evolution of the color changes (of the eight chemically treated surfaces) with the initial colorimetric values, but also with the surfaces of four control samples, which did not undergo any chemical treatment and which were exposed to the same UV radiation, under laboratory conditions.

After sampling the desired stones in Paun - Repedea village area (geographical coordinates: 47 06 $33^{\prime \prime}$ $\mathrm{N} / 27^{0} 39^{\prime} 59^{\prime \prime} \mathrm{E}$ ), the lithic material samples were obtained by cutting the stone in the form of roughly parallelepipedal slices, in order to obtain at least one flat surface for each sedimentary limestone sample.

Four of these samples, marked P0.1, P0.2, P0.3 and P0.4, are chemically untreated samples, having the role of control samples. Coated (hydrophobized) areas were delimited on the surface of each sample (of the other eight samples available for research), marked with symbols from P1 to P8 (Fig. 1).

Coating by hydrophobization of the porous limestone surface of the samples was carried out using eight chemicals for the maintenance of lithic materials, with the following trade names: LTP Mattstone H2O, LTP Colour Intensifier Stainblok Seal 2, Sikagard 700S, Sikagard 703W, Isomat Nano Pro-C, Isomat Nano-Seal, Isomat PS-20 and Tenax Ager. The hydrophobicization products used were marked with symbols from S1 to S8, being associated with the same order numbers of the symbols from P1 to P8 (Table 1). The main features and expected effects for the eight coating agents according to the technical data sheets - are shown in tables 2 and 3

The evaluation of the color changes, in the same spots on the samples and throughout the research, was performed with a Lovibond RT 300 (Reflectance Tintometer D65/10 ) spectrophotometer, using the CIE $L * a * b *$ colorimetry system, which allows the calculation of the variation of the total color change or chromatic deviation $\left(\Delta E^{*}{ }_{\mathrm{ab}}\right)$ according to the formula [13-20]:

$$
\Delta E_{a b}^{*}=\sqrt{\left(\Delta L^{*}\right)^{2}+\left(\Delta a^{*}\right)^{2}+\left(\Delta b^{*}\right)^{2}}
$$

where:

- $L^{*}$ is the change of brightness in that spot, before and after the application of hydrophobizing solutions, or before and after exposure to certain conditions, at different time intervals (n), compared to the initial (i) value: $\Delta L^{*}=L^{*}{ }_{\mathrm{n}}-L^{*}$;

- $a^{*}$ is the color change of the coordinates on the $a^{*}$ axis (from red to green), in the same spot, at different time intervals, compared to the initial value: $\Delta a^{*}=a^{*}{ }_{\mathrm{n}}-a^{*}$;

- $b^{*}$ is the color change of the coordinates on the $\mathrm{b}^{*}$ axis (from yellow to blue), by applying the same calculation method: $\Delta b^{*}=b^{*}{ }_{\mathrm{n}}-b^{*}$ (Fig. 2). 


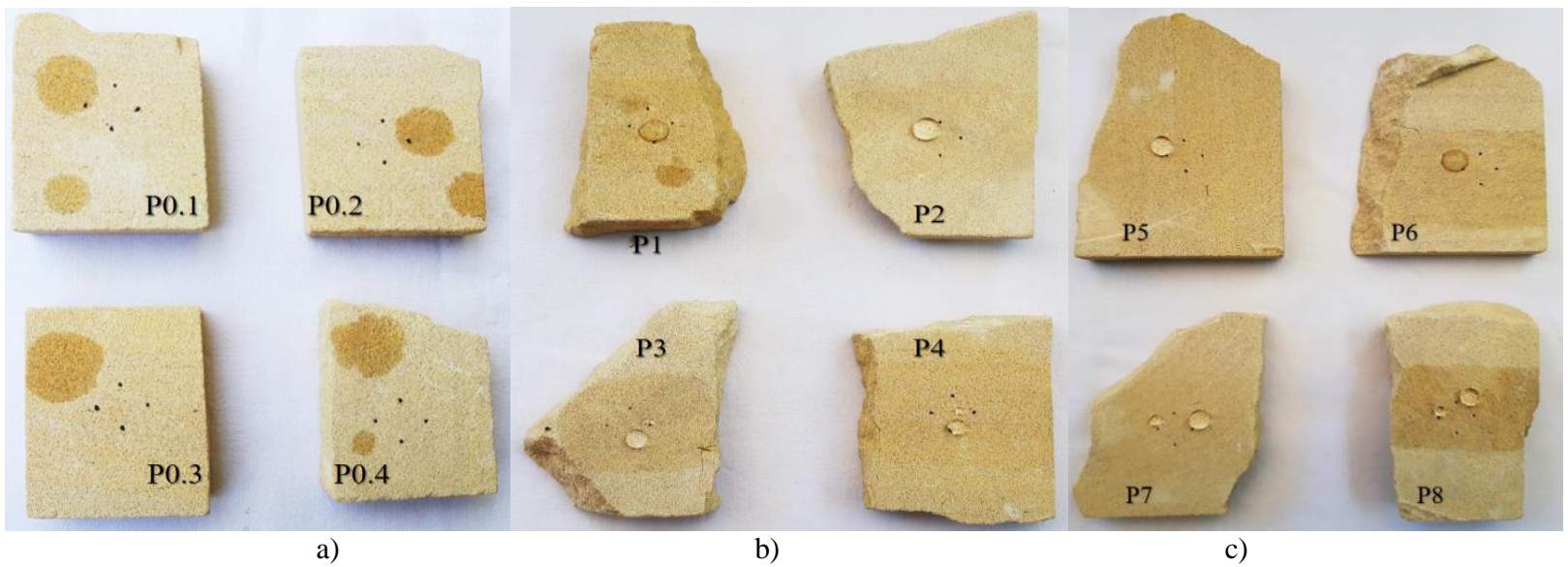

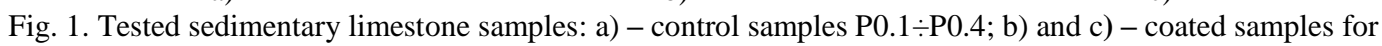
hydrophobization $\mathrm{P} 1 \div \mathrm{P} 8$

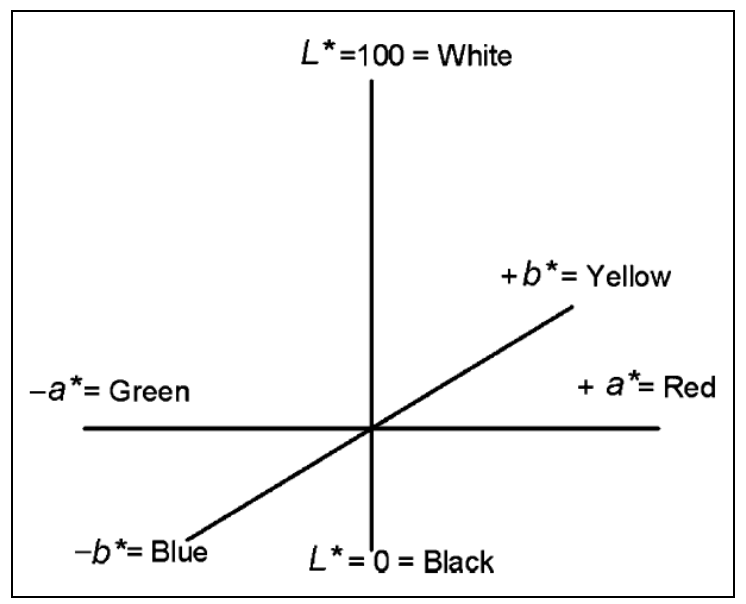

Fig. 2. Schematic representation of colorimetric coordinates $L^{*}, a^{*}, b^{*}[13]$

Exposure to UV radiation was mainly done at a wavelength of 300 to $365 \mathrm{~nm}$, using four lamps with mercury vapor under pressure (Osram ${ }^{\circledR}$ Ultra Vitalux - 300W - 230V), with the artificial stone aging standard UNI 10925/2001 as a starting point, which recommends the use of mercury vapor lamps for the simulation in time of the solar spectrum, under laboratory conditions [21-23].

The four lamps were laid out convergently with respect to the lithic samples, in a semicircle with a radius of $40 \mathrm{~cm}$, two lamps being placed in the center and the other two on the edges. Thus, all the lamps were placed at $36^{\circ}$ angle relative to the intersection point of the $40 \mathrm{~cm}$ radii, thus obtaining an increase in the number of samples, arranged linearly, that can be increasingly and simultaneously aged (Fig. 3).

For the best interpretation of the colorimetric data, a Gann Hydromette Compact B moisture meter was used noninvasively (during the monitoring of total color changes), which operates based on the principle of dielectric constant measuring by radio frequency.

After cutting, the lithic samples were stored in the desiccator for 48 hours and subsequently the following analyses (stages) were carried out:

1. initial colorimetric measurements, before coating (the data collected are summarized in table 4, except for the control samples);

2. chemical coating treatment, followed by restorage in the desiccator for another $48 \mathrm{~h}$, to stabilize the humidity of the hydrophobized surfaces;

3. colorimetric measurements to determine color changes after the chemical treatment (Table 4);

4. simultaneous UV radiation exposure of the 12 samples after hydrophobization of samples $\mathrm{P} 1 \div \mathrm{P} 8$, the total exposure period being $48 \mathrm{~h}$ for each sample. 
Table 1

SYMBOLS OF CHEMICAL HYDROPHOBICIZATION AGENTS AND OF THE TREATED SAMPLES

\begin{tabular}{|c|c|c|c|}
\hline \multirow{2}{*}{ Producer } & \multicolumn{2}{|c|}{ Chemical agents } & $\begin{array}{c}\text { Symbols of lithic } \\
\text { samples }\end{array}$ \\
\cline { 2 - 4 } & Trade names & Symbols & S1 \\
\hline \multirow{2}{*}{ LTP } & Mattstone H2O & S2 & P2 \\
\cline { 2 - 4 } & Colour Intensifier Stainblok Seal 2 & S3 & P3 \\
\hline \multirow{2}{*}{ Sika } & Sikagard 700 S & S4 & P4 \\
\cline { 2 - 4 } & Sikagard 703 W & S5 \\
\hline \multirow{2}{*}{ Isomat } & Nano Pro-C & S6 & P6 \\
\cline { 2 - 4 } & Nano-Seal & S7 & P7 \\
\hline Tenax & PS-20 & S8 & P8 \\
\hline
\end{tabular}

Table 2

GROUPING AND TECHNICAL DESCRIPTION OF CHEMICAL AGENTS USED FOR LABORATORY TESTS.ISOMAT GREECE AND SIKA ROMANIA

\begin{tabular}{|c|c|c|c|c|c|}
\hline \multirow{2}{*}{$\begin{array}{c}\text { Agent } \\
\text { producer }\end{array}$} & \multicolumn{3}{|c|}{ Isomat Greece } & \multicolumn{2}{|c|}{ Sika Romania } \\
\hline & Nanopro-C & Nano-Seal & Ps-20 & Sikagard 700S & Sikagard $703 \mathrm{~W}$ \\
\hline 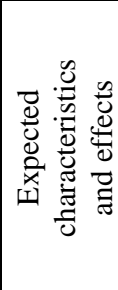 & $\begin{array}{l}\text { Aqueous dispersion, } \\
\text { in the form of an } \\
\text { emulsion, with nano- } \\
\text { molecular structure. } \\
\text { Nanoimpregnant and } \\
\text { antifungal biocide } \\
\text { for absorbing } \\
\text { surfaces. }\end{array}$ & $\begin{array}{c}\text { Resin-based } \\
\text { aqueous } \\
\text { dispersion, with } \\
\text { high penetrability, } \\
\text { due to its } \\
\text { nanomolecular } \\
\text { structure. }\end{array}$ & $\begin{array}{l}\text { Silicone emulsion with } \\
\text { solvents for } \\
\text { waterproofing } \\
\text { inorganic substrates. It } \\
\text { prevents water and } \\
\text { particulate matter } \\
\text { absorption }\end{array}$ & $\begin{array}{l}\text { Single-component } \\
\text { impregnant and } \\
\text { hydrophobic agent for } \\
\text { colorless treatment of } \\
\text { absorbent substrates. }\end{array}$ & $\begin{array}{c}\text { Hydrophobic } \\
\text { impregnating material } \\
\text { for colorless treatment of } \\
\text { absorbent substrates. } \\
\text { Biocidal agent that } \\
\text { reduces the growth of } \\
\text { fungi, algae and lichens. }\end{array}$ \\
\hline 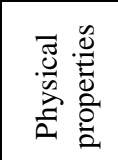 & $\begin{array}{c}\text { White emulsion with } \\
\text { specific odor, with } \\
7.5 \mathrm{pH} \text { and } 0.99 \\
\mathrm{~kg} / \mathrm{L} \text { density. }\end{array}$ & $\begin{array}{l}\text { White emulsion } \\
\text { with } \\
1.00 \mathrm{~kg} / \mathrm{L} \text { density } \\
\text { and } 8.5 \mathrm{pH} .\end{array}$ & $\begin{array}{c}\text { Transparent emulsion } \\
\text { with } 0.99 \mathrm{~kg} / \mathrm{L} \text { density. } \\
\text { Suitable for highly } \\
\text { alkaline substrates. }\end{array}$ & $\begin{array}{c}\text { Colorless liquid, based } \\
\text { on siloxane in organic } \\
\text { solvent, with } 0.800 \\
\text { kg/L density. }\end{array}$ & $\begin{array}{c}\text { Water-based silicone } \\
\text { whitish emulsion (silane } \\
+ \text { siloxane). Eco-friendly } \\
\text { material. }\end{array}$ \\
\hline
\end{tabular}

\section{Remarks:}

a) during the working stage no. 4, the colorimetric variation was checked every eight hours of exposure and for the $\mathrm{P} 1 \div \mathrm{P} 8$ areas the reference values collected after chemical treatment application were considered;

b) at the same time, the surface moisture of each analyzed area was monitored in order to determine the possible influence on colorimetric changes;

c) the values of the total color change $\Delta E^{*}$ ab collected during stage no. 4 are summarized in table 5 , whereas table 6 and 7 includes all the colorimetric values recorded during stages 1,3 and 4.

Table 3

GROUPING AND TECHNICAL DESCRIPTION OF CHEMICAL AGENTS USED FOR LABORATORY TESTS TENAX SPA ITALIA AND LTP UNITED KINGDOM

\begin{tabular}{|c|c|c|c|}
\hline Producer & Tenax Spa Italia & \multicolumn{2}{|r|}{ LTP United Kingdom } \\
\hline Agents & Ager & Mattstone $\mathrm{H} 2 \mathrm{O}$ & Colour Intensifier \& Stainblock SEAL 2 \\
\hline 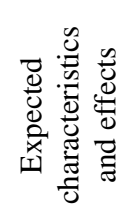 & $\begin{array}{c}\text { Hydrophobic agent } \\
\text { with major influence on } \\
\text { the appearance of color } \\
\text { after drying. }\end{array}$ & $\begin{array}{l}\text { Water-based hydrophobic } \\
\text { agent by impregnation (and } \\
\text { coating), which improves } \\
\text { the appearance of color. }\end{array}$ & $\begin{array}{l}\text { Hydrophobic agent by impregnation, for use both } \\
\text { indoors and outdoors. Suitable for all types of } \\
\text { natural and artificial stone. Provides improved } \\
\text { matte finish. Strongly protects against water, } \\
\text { grease and dirt. It allows the treated surface "to } \\
\text { breathe". }\end{array}$ \\
\hline
\end{tabular}




\begin{tabular}{|c|c|c|c|}
\hline 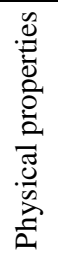 & $\begin{array}{c}\text { Colorless liquid with a } \\
\text { strong odor of organic } \\
\text { compounds, specific to } \\
\text { waxes. Commercially } \\
\text { available product } \\
\text { soluble only in organic } \\
\text { solvents. }\end{array}$ & $\begin{array}{c}\text { Colorless liquid with light } \\
\text { specific odor. Miscible with } \\
\text { water. } \\
\text { Eco-friendly material. }\end{array}$ & $\begin{array}{c}\text { Solution with strong hydrocarbon odor, } \\
\text { based on hydrocarbons (alkanes, isoalkanes, } \\
\text { aromatic hydrocarbons, naphthapetrol, etc.). Toxic } \\
\text { to the environment (especially to the aquatic } \\
\text { environment), with long lasting effects. }\end{array}$ \\
\hline
\end{tabular}

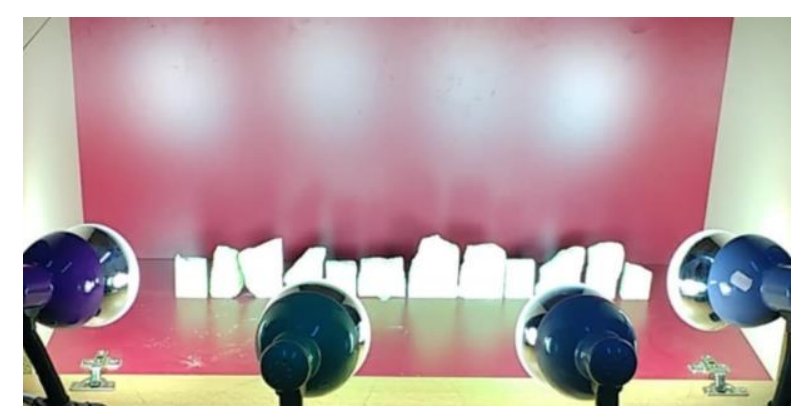

Table 4

COLORIMETRIC MEASUREMENTS FOR DETERMINING CHROMATIC CHANGES BEFORE AND AFTER THE CHEMICAL COATING TREATMENTÎ

\begin{tabular}{|c|c|c|c|c|c|c|c|}
\hline \multirow{3}{*}{ Sample } & \multicolumn{6}{|c|}{ Colorimetric values } & \multirow{3}{*}{$\begin{array}{c}\Delta E^{*} \mathrm{ab} \\
\text { value after } \\
\text { chemical } \\
\text { treatment }\end{array}$} \\
\hline & \multicolumn{3}{|c|}{ initial } & \multicolumn{3}{|c|}{ after chemical treatment } & \\
\hline & $L^{*}$ & $a^{*}$ & $b^{*}$ & $L^{*}$ & $a^{*}$ & $b^{*}$ & \\
\hline $\mathrm{P} 1$ & 74.36 & 5.20 & 20.44 & 71.15 & 6.39 & 24.46 & 5.28 \\
\hline $\mathrm{P} 2$ & 76.46 & 4.36 & 17.99 & 72.82 & 6.03 & 23.44 & 6.76 \\
\hline P3 & 74.72 & 4.04 & 17.01 & 68.73 & 5.76 & 22.82 & 8.52 \\
\hline $\mathrm{P} 4$ & 73.50 & 5.53 & 21.86 & 70.82 & 6.54 & 24.62 & 3.98 \\
\hline P5 & 72.26 & 5.89 & 23.35 & 70.87 & 6.59 & 25.68 & 2.80 \\
\hline P6 & 75.56 & 4.58 & 18.83 & 70.77 & 5.88 & 23.23 & 6.63 \\
\hline P7 & 77.25 & 4.39 & 18.88 & 75.58 & 5.18 & 21.24 & 3.00 \\
\hline P8 & 76.50 & 4.39 & 18.88 & 69.51 & 6.29 & 24.9 & 9.42 \\
\hline
\end{tabular}

Table 5

TOTAL COLOR CHANGE $\Delta E^{*}$ ab VALUES COLLECTED DURING STAGE No. 4

\begin{tabular}{|c|c|c|c|c|c|c|c|}
\hline \multirow{2}{*}{ Sample } & \multirow{2}{*}{$\begin{array}{c}\Delta E_{\text {ab value }}^{*} \\
\text { after } \\
\text { chemical } \\
\text { treatment }\end{array}$} & \multicolumn{6}{|c|}{$\begin{array}{l}\Delta E^{*} \text { ab value after UV radiation exposure, at the } \\
\text { following time intervals: }\end{array}$} \\
\hline & & $8 \mathrm{~h}$ & $16 \mathrm{~h}$ & $24 \mathrm{~h}$ & $32 \mathrm{~h}$ & $40 \mathrm{~h}$ & $48 \mathrm{~h}$ \\
\hline P0.1 & - & 0.37 & 0.81 & 0.69 & 0.82 & 0.95 & 0.50 \\
\hline P0.2 & - & 0.27 & 0.21 & 0.15 & 0.33 & 0.22 & 0.25 \\
\hline P0.3 & - & 0.64 & 0.98 & 0.79 & 1.18 & 1.23 & 1.60 \\
\hline P0.4 & - & 0.66 & 1.16 & 0.57 & 0.56 & 0.78 & 0.63 \\
\hline $\mathrm{P} 1$ & 5.28 & 0.60 & 0.65 & 0.49 & 0.38 & 0.32 & 0.46 \\
\hline $\mathrm{P} 2$ & 6.76 & 1.78 & 1.86 & 2.23 & 2.57 & 2.79 & 2.84 \\
\hline P3 & 8.52 & 1.90 & 2.09 & 2.25 & 2.51 & 2.56 & 2.54 \\
\hline $\mathrm{P} 4$ & 3.98 & 0.67 & 0.88 & 1.22 & 1.39 & 1.49 & 0.87 \\
\hline P5 & 2.80 & 1.14 & 1.45 & 1.33 & 1.56 & 1.82 & 1.86 \\
\hline P6 & 6.63 & 0.92 & 0.98 & 1.00 & 1.19 & 1.04 & 0.93 \\
\hline P7 & 3.00 & 0.74 & 0.99 & 1.14 & 1.29 & 1.30 & 1.10 \\
\hline P8 & 9.42 & 1.29 & 1.50 & 1.77 & 2.00 & 2.06 & 2.19 \\
\hline
\end{tabular}


Table 6

COLORIMETRIC VALUES RECORDED FOR SAMPLES P0.1 $\div$ P0.4,

DURING THE WORKING STAGES No. 1 AND 4

\begin{tabular}{|c|c|c|c|c|c|c|}
\hline \multirow{2}{*}{ Sample } & \multirow{2}{*}{\multicolumn{2}{|c|}{ Working stage }} & \multicolumn{3}{|c|}{ CIE colorimetric coordinates } & \multirow{2}{*}{$\begin{array}{l}\text { Total color change } \\
\text { index } \Delta E^{*} \mathrm{ab}\end{array}$} \\
\hline & & & $L^{*}$ & $a^{*}$ & $b^{*}$ & \\
\hline \multirow{7}{*}{ P0.1 } & \multicolumn{2}{|c|}{ CIE values in the initial stage* } & 73.66 & 5.60 & 22.15 & - \\
\hline & \multirow{6}{*}{$\begin{array}{l}\text { CIE values } \\
\text { after UV } \\
\text { radiation } \\
\text { exposure, at } \\
8 \mathrm{~h} \\
\text { intervals** }\end{array}$} & $8 \mathrm{~h}$ & 73.64 & 5.39 & 21.84 & 0.37 \\
\hline & & $16 \mathrm{~h}$ & 73.79 & 5.32 & 21.40 & 0.81 \\
\hline & & $24 \mathrm{~h}$ & 73.86 & 5.25 & 21.59 & 0.69 \\
\hline & & $32 \mathrm{~h}$ & 73.86 & 5.26 & 21.43 & 0.82 \\
\hline & & $40 \mathrm{~h}$ & 73.93 & 5.27 & 21.30 & 0.95 \\
\hline & & $48 \mathrm{~h}$ & 73.65 & 5.30 & 21.75 & 0.50 \\
\hline \multirow{7}{*}{ P0.2 } & \multicolumn{2}{|c|}{ CIE values in the initial stage* } & 73.38 & 5.13 & 20.46 & - \\
\hline & \multirow{6}{*}{$\begin{array}{l}\text { CIE values } \\
\text { after UV } \\
\text { radiation } \\
\text { exposure, at } \\
\quad 8 \mathrm{~h} \\
\text { intervals** }\end{array}$} & $8 \mathrm{~h}$ & 73.28 & 5.01 & 20.24 & 0.27 \\
\hline & & $16 \mathrm{~h}$ & 73.35 & 4.99 & 20.30 & 0.21 \\
\hline & & $24 \mathrm{~h}$ & 73.53 & 5.02 & 20.25 & 0.15 \\
\hline & & $32 \mathrm{~h}$ & 73.54 & 4.96 & 20.22 & 0.33 \\
\hline & & $40 \mathrm{~h}$ & 73.44 & 5.01 & 20.29 & 0.22 \\
\hline & & $48 \mathrm{~h}$ & 73.52 & 4.94 & 20.37 & 0.25 \\
\hline \multirow{7}{*}{ P0.3 } & \multicolumn{2}{|c|}{ CIE values in the initial stage* } & 72.44 & 6.08 & 23.64 & - \\
\hline & \multirow{6}{*}{$\begin{array}{l}\text { CIE values } \\
\text { after UV } \\
\text { radiation } \\
\text { exposure, at } \\
\text { 8h intervals* }\end{array}$} & $8 \mathrm{~h}$ & 72.54 & 5.77 & 23.09 & 0.64 \\
\hline & & $16 \mathrm{~h}$ & 72.94 & 5.74 & 22.87 & 0.98 \\
\hline & & $24 \mathrm{~h}$ & 72.82 & 5.87 & 22.98 & 0.79 \\
\hline & & $32 \mathrm{~h}$ & 73.17 & 5.67 & 22.81 & 1.18 \\
\hline & & $40 \mathrm{~h}$ & 73.10 & 5.70 & 22.67 & 1.23 \\
\hline & & $48 \mathrm{~h}$ & 73.83 & 5.69 & 22.96 & 1.60 \\
\hline \multirow{7}{*}{ P0.4 } & \multicolumn{2}{|c|}{ CIE values in the initial stage* } & 76.44 & 4.88 & 20.19 & - \\
\hline & \multirow{6}{*}{$\begin{array}{c}\text { CIE values } \\
\text { after UV } \\
\text { radiation } \\
\text { exposure, at } \\
8 \mathrm{~h} \\
\text { intervals** }\end{array}$} & $8 \mathrm{~h}$ & 76.16 & 4.69 & 19.62 & 0.66 \\
\hline & & $16 \mathrm{~h}$ & 75.45 & 4.70 & 19.62 & 1.16 \\
\hline & & $24 \mathrm{~h}$ & 76.35 & 4.75 & 19.64 & 0.57 \\
\hline & & $32 \mathrm{~h}$ & 76.33 & 4.66 & 19.69 & 0.56 \\
\hline & & $40 \mathrm{~h}$ & 76.25 & 4.63 & 19.48 & 0.78 \\
\hline & & $48 \mathrm{~h}$ & 76.39 & 4.61 & 19.62 & 0.63 \\
\hline
\end{tabular}

*Stages 1 and **Stages 4

Table 7

COLORIMETRIC VALUES RECORDED FOR SAMPLES P $1 \div \mathrm{P} 8$

DURING THE WORKING STAGES No. 1, 3 AND 4 (*Stages 1 , **Stages 3 and ***Stages 4)

\begin{tabular}{|c|c|c|c|c|c|c|}
\hline \multirow{2}{*}{ Sample } & \multirow{2}{*}{\multicolumn{2}{|c|}{ Working stage }} & \multicolumn{3}{|c|}{ CIE colorimetric coordinates } & \multirow{2}{*}{$\begin{array}{l}\text { Total color change } \\
\text { index } \Delta E^{*}{ }_{\mathrm{ab}}\end{array}$} \\
\hline & & & $L^{*}$ & $a^{*}$ & $b^{*}$ & \\
\hline \multirow{8}{*}{$\mathrm{P} 1$} & \multicolumn{2}{|c|}{ CIE values in the initial stage* } & 74.36 & 5.20 & 20.44 & - \\
\hline & \multicolumn{2}{|c|}{$\begin{array}{c}\text { CIE values after chemical } \\
\text { treatment } * *\end{array}$} & 71.15 & 6.39 & 24.46 & 5.28 \\
\hline & \multirow{6}{*}{$\begin{array}{c}\text { CIE values } \\
\text { after UV } \\
\text { radiation } \\
\text { exposure, at } \\
\text { 8h } \\
\text { intervals } * * *\end{array}$} & $8 \mathrm{~h}$ & 70.63 & 6.30 & 24.75 & 0.60 \\
\hline & & $16 \mathrm{~h}$ & 70.68 & 6.37 & 24.91 & 0.65 \\
\hline & & $24 \mathrm{~h}$ & 70.83 & 6.38 & 24.83 & 0.49 \\
\hline & & $32 \mathrm{~h}$ & 70.97 & 6.17 & 24.71 & 0.38 \\
\hline & & $40 \mathrm{~h}$ & 70.93 & 6.18 & 24.57 & 0.32 \\
\hline & & $48 \mathrm{~h}$ & 70.93 & 5.99 & 24.39 & 0.46 \\
\hline \multirow{8}{*}{$\mathrm{P} 2$} & \multicolumn{2}{|c|}{ CIE values in the initial stage* } & 76.46 & 4.36 & 17.99 & - \\
\hline & \multicolumn{2}{|c|}{$\begin{array}{c}\text { CIE values after chemical } \\
\text { treatment } * *\end{array}$} & 72.82 & 6.03 & 23.44 & 6.76 \\
\hline & \multirow{6}{*}{$\begin{array}{l}\text { CIE values } \\
\text { after UV } \\
\text { radiation } \\
\text { exposure, at } \\
8 \mathrm{~h} \\
\text { intervals*** }\end{array}$} & $8 \mathrm{~h}$ & 73.55 & 5.36 & 21.96 & 1.78 \\
\hline & & $16 \mathrm{~h}$ & 73.96 & 5.46 & 22.09 & 1.86 \\
\hline & & $24 \mathrm{~h}$ & 73.78 & 5.25 & 21.59 & 2.23 \\
\hline & & $32 \mathrm{~h}$ & 73.95 & 5.07 & 21.34 & 2.57 \\
\hline & & $40 \mathrm{~h}$ & 74.09 & 5.04 & 21.16 & 2.79 \\
\hline & & $48 \mathrm{~h}$ & 74.05 & 4.94 & 21.12 & 2.84 \\
\hline
\end{tabular}




\begin{tabular}{|c|c|c|c|c|c|c|}
\hline \multirow{8}{*}{ P3 } & \multirow{2}{*}{\multicolumn{2}{|c|}{$\begin{array}{c}\text { CIE values in the initial stage* } \\
\text { CIE values after chemical } \\
\text { treatment } * *\end{array}$}} & 74.72 & 4.04 & 17.01 & - \\
\hline & & & 68.73 & 5.76 & 22.82 & 8.52 \\
\hline & \multirow{6}{*}{$\begin{array}{l}\text { CIE values } \\
\text { after UV } \\
\text { radiation } \\
\text { exposure, at } \\
8 \mathrm{~h} \\
\text { intervals*** }\end{array}$} & $8 \mathrm{~h}$ & 70.07 & 5.24 & 21.58 & 1.90 \\
\hline & & $16 \mathrm{~h}$ & 70.33 & 5.18 & 21.61 & 2.09 \\
\hline & & $24 \mathrm{~h}$ & 70.34 & 5.17 & 21.36 & 2.25 \\
\hline & & $32 \mathrm{~h}$ & 70.60 & 4.97 & 21.34 & 2.51 \\
\hline & & $40 \mathrm{~h}$ & 70.59 & 4.98 & 21.24 & 2.56 \\
\hline & & $48 \mathrm{~h}$ & 70.51 & 4.96 & 21.19 & 2.54 \\
\hline \multirow{8}{*}{ P4 } & \multicolumn{2}{|c|}{ CIE values in the initial stage* } & 73.50 & 5.53 & 21.86 & - \\
\hline & \multicolumn{2}{|c|}{$\begin{array}{c}\text { CIE values after chemical } \\
\text { treatment } * *\end{array}$} & 70.82 & 6.54 & 24.62 & 3.98 \\
\hline & \multirow{6}{*}{$\begin{array}{c}\text { CIE values } \\
\text { after UV } \\
\text { radiation } \\
\text { exposure, at } \\
8 \mathrm{~h} \\
\text { intervals*** }\end{array}$} & $8 \mathrm{~h}$ & 71,00 & 6.24 & 24.05 & 0.67 \\
\hline & & $16 \mathrm{~h}$ & 71.16 & 6.26 & 23.86 & 0.88 \\
\hline & & $24 \mathrm{~h}$ & 71.39 & 6.15 & 23.62 & 1.22 \\
\hline & & $32 \mathrm{~h}$ & 71.46 & 5.96 & 23.53 & 1.39 \\
\hline & & $40 \mathrm{~h}$ & 71.44 & 5.99 & 23.38 & 1.49 \\
\hline & & $48 \mathrm{~h}$ & 71.46 & 5.89 & 23.40 & 0.87 \\
\hline \multirow{8}{*}{ P5 } & \multicolumn{2}{|c|}{ CIE values in the initial stage* } & 72.26 & 5.89 & 23.35 & - \\
\hline & \multicolumn{2}{|c|}{$\begin{array}{c}\text { CIE values after chemical } \\
\text { treatment } * *\end{array}$} & 70.87 & 6.59 & 25.68 & 2.80 \\
\hline & \multirow{6}{*}{$\begin{array}{l}\text { CIE values } \\
\text { after UV } \\
\text { radiation } \\
\text { exposure, at } \\
8 \mathrm{~h} \\
\text { intervals*** }\end{array}$} & $8 \mathrm{~h}$ & 70.73 & 6.21 & 24.61 & 1.14 \\
\hline & & $16 \mathrm{~h}$ & 71.95 & 6.26 & 24.77 & 1.45 \\
\hline & & $24 \mathrm{~h}$ & 71.42 & 6.24 & 24.52 & 1.33 \\
\hline & & $32 \mathrm{~h}$ & 71.44 & 6.08 & 24.32 & 1.56 \\
\hline & & $40 \mathrm{~h}$ & 71.01 & 6.01 & 23.96 & 1.82 \\
\hline & & $48 \mathrm{~h}$ & 71.40 & 5.94 & 24.02 & 1.86 \\
\hline \multirow{8}{*}{ P6 } & \multicolumn{2}{|c|}{ CIE values in the initial stage* } & 75.56 & 4.58 & 18.83 & - \\
\hline & \multicolumn{2}{|c|}{$\begin{array}{c}\text { CIE values after chemical } \\
\text { treatment } * *\end{array}$} & 70.77 & 5.88 & 23.23 & 6.63 \\
\hline & \multirow{6}{*}{$\begin{array}{l}\text { CIE values } \\
\text { after UV } \\
\text { radiation } \\
\text { exposure, at } \\
8 \mathrm{~h} \\
\text { intervals*** }\end{array}$} & $8 \mathrm{~h}$ & 71.28 & 5.44 & 22.60 & 0.92 \\
\hline & & $16 \mathrm{~h}$ & 71.35 & 5.44 & 22.58 & 0.98 \\
\hline & & $24 \mathrm{~h}$ & 71.35 & 5.45 & 22.54 & 1.00 \\
\hline & & $32 \mathrm{~h}$ & 71.86 & 5.42 & 23.10 & 1.19 \\
\hline & & $40 \mathrm{~h}$ & 71.36 & 5.36 & 22.55 & 1.04 \\
\hline & & $48 \mathrm{~h}$ & 71.36 & 5.34 & 22.76 & 0.93 \\
\hline \multirow{8}{*}{ P7 } & \multicolumn{2}{|c|}{ CIE values in the initial stage* } & 77.25 & 4.39 & 18.88 & - \\
\hline & \multicolumn{2}{|c|}{$\begin{array}{l}\text { CIE values after chemical } \\
\text { treatment } * *\end{array}$} & 75.58 & 5.18 & 21.24 & 3.00 \\
\hline & \multirow{6}{*}{$\begin{array}{l}\text { CIE values } \\
\text { after UV } \\
\text { radiation } \\
\text { exposure, at } \\
8 \mathrm{~h} \\
\text { intervals*** }\end{array}$} & $8 \mathrm{~h}$ & 75.84 & 4.88 & 20.61 & 0.74 \\
\hline & & $16 \mathrm{~h}$ & 75.90 & 4.81 & 20.38 & 0.99 \\
\hline & & $24 \mathrm{~h}$ & 76.10 & 4.82 & 20.29 & 1.14 \\
\hline & & $32 \mathrm{~h}$ & 76.18 & 4.70 & 20.21 & 1.29 \\
\hline & & $40 \mathrm{~h}$ & 76.17 & 4.71 & 20.18 & 1.30 \\
\hline & & $48 \mathrm{~h}$ & 76.24 & 4.72 & 20.49 & 1.10 \\
\hline \multirow{8}{*}{ P8 } & \multicolumn{2}{|c|}{ CIE values in the initial stage* } & 76.50 & 4.39 & 18.88 & - \\
\hline & \multicolumn{2}{|c|}{$\begin{array}{c}\text { CIE values after chemical } \\
\text { treatment } * *\end{array}$} & 69.51 & 6.29 & 24.90 & 9.42 \\
\hline & & $8 \mathrm{~h}$ & 70.61 & 5.97 & 24.31 & 1.29 \\
\hline & after UV & $16 \mathrm{~h}$ & 70.82 & 5.94 & 24.25 & 1.50 \\
\hline & radiation & $24 \mathrm{~h}$ & 71.00 & 5.90 & 24.02 & 1.77 \\
\hline & exposure, at & $32 \mathrm{~h}$ & 71.08 & 5.71 & 23.81 & 2.00 \\
\hline & $8 \mathrm{~h}$ & $40 \mathrm{~h}$ & 71.08 & 5.69 & 23.71 & 2.06 \\
\hline & intervals $* * *$ & $48 \mathrm{~h}$ & 71.35 & 5.69 & 23.88 & 2.19 \\
\hline
\end{tabular}

\section{Results and discussions}

As one may notice, the photographs in figure 1 were taken prior to accelerated aging by UV radiation exposure, by previously using the drip method to check surfaces in relation to water drops. As far as control samples are concerned (Fig. 1a), the strong hydrophilic nature of the porous rocks in the Paun-Repedea area is noticed and, as for samples P1 to P8 (Fig. 1b and c), the hydrophobic character of the permeabilized surfaces and implicitly of the eight solutions used is confirmed. 
Considering the most recent studies and researches on the total color change $\Delta E^{*}$ ab values in the CIE $L^{*} a^{*} b^{*}$ colorimetry system, most authors argue that the minimal significance threshold of color changes (perceptible by the human eye) is at least 5 units, which means that the $\Delta E^{*}$ ab value will be at least equal to or greater than $5\left(\Delta E^{*}{ }_{a b} \geq 5\right)$ [13], although prior to 2007 other authors set the minimum threshold of the $\Delta E^{*}$ ab value (for a noticeable color change) to 2.5 , in the same CIE $L * a * b *$ system [24].

Nevertheless, some authors specialized in the assessment of consolidated, impregnated or coated lithic surfaces actually use the $\Delta E^{*}$ ab Scale in Stone Materials Conservation which provides the following interpretation of the total color change [25]:

1. $\Delta E^{*}{ }_{\mathrm{ab}}<0.2$ : no perceivable difference;

2. $0.2<\Delta E^{*}$ ab $<0.5$ : very small difference;

3. $\quad 0.5<\Delta E^{*}{ }_{\mathrm{ab}}<2$ : small difference;

4. $2<\Delta E^{*}$ ab $<3$ : fairly perceptible difference;

5. $3<\Delta E_{\mathrm{ab}}^{*}<6$ : perceptible difference;

6. $6<\Delta E_{\mathrm{ab}}^{*}<12$ : strong difference;

7. $\Delta E^{*}{ }_{\mathrm{ab}}>12$ : different color.

Considering the data in the paragraphs above and analyzing the values in table 5 , we may conclude that after chemical hydrophobicization treatment samples P1, P2, P3, P6 and P8 "underwent" a $\Delta E^{*}{ }_{\mathrm{ab}}$ color change greater than 5 units, which means that the $\mathrm{S} 1$ - LTP Mattstone $\mathrm{H} 2 \mathrm{O}$ solution causes a noticeable difference $\left(\Delta E^{*}{ }_{\mathrm{ab}}=5.28\right)$ and the products $\mathrm{S} 2$, S3, S6 and S8 show major color differences, as follows:

1. S2 - LTP Colour Intensifier Stainblok Seal $2\left(\Delta E^{*}{ }_{\mathrm{ab}}=6.76\right)$;

2. $\mathrm{S} 3$ - Sikagard 700S $\left(\Delta E_{\mathrm{ab}}^{*}=8.52\right)$

3. S6 - Isomat Nano -Seal $\left(\Delta E_{\mathrm{ab}}=6.63\right)$;

4. $\mathrm{S} 8$ - Tenax Ager $\left(\Delta E^{*} \mathrm{ab}=9.42\right)$.

After coating, the rest of the solutions (S4, S5 and S7) cause small perceptible differences, a desirable aspect especially in the case of the Science of Heritage Immovable Property Conservation [25].

In contrast, after UV radiation exposure, one may notice that both the control samples and the chemically treated samples do not show major color changes, visible to the naked eye, as the recorded values are below the minimum significance threshold of 5 units. Thus, the $\Delta E^{*}{ }_{a b}$ variations of the control samples $\mathrm{P} 0.1 \div \mathrm{P} 0.4$ are shown as follows:

- after $24 \mathrm{~h}$ - the values range between 0.15 (P0.2) and 0.79 (P0.3);

- after $48 \mathrm{~h}$ - the values range between 0.25 (P0.2) and 1.60 (P0.3).

As far as UV radiation exposure is concerned, please note that samples P2 and P3 - chemically treated - are the only ones showing "slight" $\Delta E^{*}$ ab index variations, which may be included in the fairly perceptible difference range:

- after $24 \mathrm{~h}-\Delta E_{\mathrm{ab}}^{*} \mathrm{P} 2=2.23$ and $\Delta E_{\mathrm{ab}}^{*} \mathrm{P} 3=2.25$

- after $48 \mathrm{~h}-\Delta E^{*}{ }_{\mathrm{ab}} \mathrm{P} 2=2.84$ and $\Delta E^{*}{ }_{\mathrm{ab}} \mathrm{P} 3=2.54$.

Also, a fairly perceptible difference due to UV radiation exposure may also be noticed in sample P8 but only after 48 hours of irradiation, when the $\Delta \mathrm{E}^{*} \mathrm{ab}$ index reaches the value of 2.19 units.

We would also like to point out that during the $48 \mathrm{~h}$ of UV radiation exposure, the coated samples P1, P4, P5, P6 and P7 show $\Delta E^{*}{ }_{a b}<2$ index values, which means that the agents used exhibit better resistance to UV rays.

An important aspect highlighted during these analyses is the fact that all four control samples chemically untreated but exposed to the same UV radiation recorded very small and insignificant total color changes, the $\Delta E^{*}{ }_{a b}$ values being at the end of the $48 \mathrm{~h}$ exposure below the threshold of 2 units, which means that by hydrophobic coating of natural stones in Iași area, there is the risk of color changes, especially in relation to the increasingly "aggressive" solar radiation over the last decades.

\section{Conclusions}

Considering that for the restoration and/or preservation of historical buildings any action must be as reversible as possible and to approach the architecture, shape and initial appearance (including colors of apparent materials), a prior testing of any coating material is required [26]. Therefore, if we analyze the results of the hydrophobization tests and subsequently of UV radiation under laboratory conditions, of the eight agents our attention was drawn by S4, S5 and S7 (Sikagard $703 \mathrm{~W}$, Isomat Nano Pro-C and Isomat PS-20, respectively), the $\Delta E^{*}{ }_{\mathrm{ab}}$ values of which after the initial treatment are $<5$ and the recorded total color change values after UV exposure are insignificant.

Considering the following aspects:

a) fast development of the construction sector in Romania over the last ten years; 
b) increasingly wider range of high-performance materials and products in the construction sector, through all sales and marketing channels (from manufacturers, importers and even the smallest specialty shops), including the everincreasing number of hydrophobization products;

c) poorer interest in historic buildings preservation, which requires a different approach compared to any civil building;

d) dynamics of Romanian standards in the Construction Sector in recent years, which do not cover many issues from the viewpoint of natural stone preservation in historical buildings, such as those in other countries of the European Union;

e) the poor research conducted in Romania, that would develop and harmonize romanian standards with the European and international working standards described in the Conservation Science of Heritage Immovable Property,

this paper it may be considered as a preliminary step towards a modern and innovative approach in the field of performance assessment of commercially available coating and hydrophobization products for natural stone, including in the case of the small budget of a laboratory concerned with this topic.

Last but not least, one should note that the applicability of the preliminary assessment methods described in this paper may be useful in the construction sector, and especially in the preservation of historical monuments, where preliminary and/or permanent analyses and investigations of all materials are required, in order to avoid various incompatibilities or undesirable and irreversible results.

In the case of civil buildings, each beneficiary will decide on its own behalf the type of hydrophobization agent chosen to coat indigenous natural stones in the Iași area, depending on the interpretation of the laboratory results but also on the personal options, regarding the aesthetics and the final appearance for apparent lithic surfaces.

Acknowledgment:This work was co-funded by the European Social Fund, through Operational Programme Human Capital 2014-2020, project number POCU/380/6/13/123623, project title < $<$ PhD Students and Postdoctoral Researchers Prepared for the Labour Market! >>Also, this research was supported by the Romanian Space Agency (ROSA) within Space Technology and Advanced Research (STAR) Program, Project no. 169/20.07.2017.

\section{References}

1. CAZACU, M.M., TUDOSE, O., BOSCORNEA, A., BUZDUGAN, L., TIMOFTE, A., NICOLAE, D., , Romanian Reports in Physics 69, 2017, p. 1.

2. BANICA, A., BOBRIC, E.D., CAZACU, M.M., TIMOFTE, A., GURLUI, S., BREABAN, I.G., Environmental Engineering and Management Journal, 16, 2017, p. 2147

3. SFICA, L., IORDACHE, I., ICHIM, P., LEAHU, A., CAZACU, M.M., GURLUI, S., TRIF, C.R., Present Environment and Sustainable Development, 12, 2018, p. 47.

4. BADARAU, D., CAPROSU, I., Iasi - city of the old buildings: until 1821 (in romanian language), Demiurg Publishing, Iaşi, Romania, 2007, p. 63.

5. GRASU, C., BRANZILA, M., MICLAUS, C., BOBOS, I., The Sarmatian from the System of Foreland Basins of the Eastern Carpathians (in romanian language), Universitaria Collection, Tehnical Publishing, Bucharest, Romania, 2002.

6. MUTIHAC, V., Geological Structure of the Territory of Romania (in romanian language), Tehnical Publishing, Bucharest, Romania, 1990.

7. BELL, F.G., Environmental Geology, 21, no. 4, 1993, p. 187.

8. PAUNEL, E., Contributions to the Study of Adhesion Between Romanian Cement and Indigenous Rocks, PhD thesis, Gheorghe Asachi Technical University of Iasi, Faculty of Civil Engineering and Building Services, 1970.

9. PELIN, V., RATOI, B., COVATARIU, D., SANDU, I.G., BRANZILA, M., CIOCAN, V., RADINSCHI, I., SANDU, I., Studies on the Characteristics of Calcareous Sedimentary Rocks from Iasi County, with Applicability in the Restoration of Historical Monuments, proceedings of the Xth European Exhibition Creativity and Innovation EUROINVENT - Workshop Scientific, Technological and Innovative Research in Current European Context, PIM Publishing, Iaşi, Romania, 2018, p. 25.

10. SMITH, M.R., Stone: Building Stone, Rock Fill and Armourstone in Construction, Geological Society, Special Publication, vol. 16, editor: Smith, M.R, The Geological Society Publishing House, London, UK, 1999, p. 52.

11. SANDU, I., BRANZILA, M, SANDU, I.G., Scientific Preservation of Stone Monuments (in romanian language), Alexandru Ioan Cuza University Publishing, Iași, 2009.

12. FELLER, R., Bulletin de l’lnstitut Royal du Patrimoine Artistique, XV, 1975, p. 135.

13. SCHANDA, J., Colorimetry. Understanding the CIE System, Wiley Interscience Publisher, Hoboken, New Jersey, USA, 2007.

14. SAVIUC-PAVAL, A.M., SANDU, I., POPA, I.M., SANDU, I.C.A., VASILACHE, V., SANDU, I.G., Rev. Chim. (Bucharest), 63, no. 2, 2012, p. 170.

15. PELIN, V., SANDU, I., MUNTEANU, M., IURCOVSCHI, C.T., GURLUI, S., SANDU, A.V., VASILACHE, V., BRANZILA, M., SANDU, I.G., Colour Change Evaluation on UV Radiation Exposure for Paun-Repedea Calcareous Geomaterial, proceedings in International Conference on Innovative Research - ICIR Euroinvent, edited by: Sandu, A.V., Abdullah, M.M.B., Vizureanu, P., AbdRahim, S.Z., Sandu, I., Book Series: IOP Conference Series-Materials Science and Engineering, 133, 2016, Article Number: 012061, DOI: 10.1088/1757-899X/133/1/012061

16. PELIN, V., SANDU, I., GURLUI, S., BRANZILA, M., VASILACHE, V., BORS, E., SANDU, I.G., Color Research and Application, 41, no. 3, 2016, p. 317.

17. PELIN, V., SANDU, I., GURLUI, S., BRANZILA, M., VASILACHE, V., SANDU, I.G., Rev. Chim. (Bucharest), 67, no.12, 2016, p. 2568. 18. LUVIDI, L., MECCHI, A.M., FERRETTI, M., SIDOTI, G., International Journal of Conservation Science, 7, 2016 , p. 311 , Special Issue 1. 
19. HELMI, F.M., HEFNI, Y.K., International Journal of Conservation Science, 7, no. 1, 2016, p. 29.

20. GROSSI, D., DEL LAMA, E.A., GARCIA-TALEGON, J., INIGO, A.C., VICENTE-TAVERA, S., International Journal of Conservation Science, 6, no. 3, 2015, p. 313.

21. LA RUSSA, M.F., BARONE, G., BELFIORE, C.M., MAZZOLENI, P., PEZZINO, A., Environmental Earth Sciences, 62, 2011 , p. 1263.

22. BARONE, G., CAMPANI, E., CASOLI, A., LA RUSSA, M.F., LO GIUDICE, A., MAZZOLENI, P., PEZZINO, A., Environmental Geology, 54, 2008, 1501-1506.

23. *** UNI 10925:2001 Beni culturali - Materiali lapidei naturali ed artificiali - Metodologia per l'irraggiamento con luce solare artificiale (Cultural heritage - Natural and Artificial Stone Materials - Methodology for Radiation with Artificial Sunlight), Italy.

24. SHARMA, G., Digital Color Imaging Handbook, CRC Press, 2003.

25. ALDOASRI, M.A., DARWISH, S.S., ADAM, A.A., ELMARZUGI, N.A., AHMED, S.M., Sustainability, 9, no.11, 2017, Article Number: 2002, doi.org/10.3390/su9112002

26. JOKILEHTO, J., Conservation and Management of Archaeological Sites, 2, 1998, p. 229.

$\overline{\text { Manuscript received: } 20.12 .2019}$ 